\title{
Evaluation of the translational free energy in a melting temperature calculation by simulation
}

\author{
Pankaj A. Apte and Isamu Kusaka \\ The Koffolt Laboratories, The Department of Chemical and Biomolecular Engineering, The Ohio State University, \\ 140 West 19th Avenue, Columbus, Ohio 43210, USA \\ (Received 17 May 2005; revised manuscript received 29 November 2005; published 25 January 2006)
}

\begin{abstract}
We present two methods suitable for controlling the translational degrees of freedom of a system when evaluating directly the free energy difference between the liquid and the solid phases by thermodynamic integration along a reversible path connecting these two phases. Such a constraint is crucial for an accurate prediction of the melting point by means of simulation. In one of the methods, the free energy difference was calculated by fixing one of the particles of the system at the center of the simulation box. In the second method, the free energy difference was calculated by constraining the center of mass of the system to a small region taken around the center of the simulation box. The correction to the free energy difference due to each constraint must be evaluated by a direct simulation. Both methods give consistent results when applied to a truncated and shifted Lennard-Jones system with cutoff radius of $2.5 \sigma$. However, the fixed particle constraint method is found to be more efficient computationally.
\end{abstract}

DOI: 10.1103/PhysRevE.73.016704

PACS number(s): 05.10.-a, 05.20.-y, 64.70.Dv

\section{INTRODUCTION}

A crucial step in determining the melting temperature of a model system by simulation is to obtain the absolute free energy of the solid phase by thermodynamic integration along a pathway that connects the solid phase at a state of interest to a reference state of known free energy [1]. The pathway is usually characterized by a single parameter $\lambda$ controlling the potential energy $\phi$ of the system along the path by a formula such as

$$
\phi(\lambda)=\lambda U+(1-\lambda) U_{e x t},
$$

where $U$ is the potential energy due to interaction among the particles and $U_{e x t}$ is some properly chosen external potential field. As $\lambda$ varies from 0 to 1 , the state of the system changes from the reference state to the desired solid state and the Helmholtz free energy change along the path is given by

$$
\Delta F=\int_{0}^{1} d \lambda\left\langle\frac{\partial \phi}{\partial \lambda}\right\rangle_{T, V, N} .
$$

For this approach to be successful, there should be no firstorder phase transition along the path. In addition, the integrand in Eq. (2) must be well behaved, i.e., (i) the integrand should not vary sharply with $\lambda$ and (ii) the statistical error in evaluating the integrand by simulation should be as small as possible. To fulfill these requirements, one must often restrict the translational degrees of freedom of the system, for example, by restricting its center of mass [1-4]. However, the change in the free energy resulting from this constraint is not always carefully accounted for with the notable exception of the Einstein crystal method [1,2], in which the correction term is given analytically. In this article, we focus on the recently introduced thermodynamic integration scheme of Grochola [4] and develop two simple methods to constrain the translational degrees of freedom of the system suitable for this approach.

Among the class of thermodynamic integration techniques, the method proposed in Ref. [4] is unique in that it directly calculates the Helmholtz free energy difference between the solid phase and the liquid phase. This is to be contrasted with the previous techniques $[1-3,5]$, in which the absolute free energies of the liquid and the solid phases need to be computed separately in order to calculate the free energy difference between the two phases. The other class of methods that directly calculates the free energy difference between the solid and the liquid phases is the phase switch Monte Carlo method [6] which has recently been extended to Lennard-Jones systems [7]. For an overview of other simulation approaches for calculating the melting temperature, we refer the reader to Refs. $[7,8]$. The method presented in Ref. [4] appears promising. For example, it has recently been applied to calculate the melting temperature of sodium chloride [8] and to calculate the surface tension of the $\mathrm{Au}(100)$ surface [9]. For this method, however, a rigorous formulation has not been proposed to account for the change in free energy resulting from the constraint on the translational degrees of freedom of the system. The approach developed by Frenkel and Ladd [1,2] for this purpose is suitable for the Einstein crystal method, but becomes prohibitively expensive when applied in Grochola's method. We address this problem in the present work.

In many thermodynamic integration methods, the Gibbs free energy difference $\Delta G=G_{S}-G_{L}$ between the solid and the liquid phases is calculated indirectly, i.e., one first calculates the Helmholtz free energy difference $\Delta F=F_{S}-F_{L}$, from which $\Delta G$ is obtained by using the relation $\Delta G=\Delta F+P\left(\langle V\rangle_{S}-\langle V\rangle_{L}\right)$, where $\langle V\rangle_{S}$ and $\langle V\rangle_{L}$ are the average volumes of the solid and the liquid phases at given temperature $T$, pressure $P$, and number of particles $N$. In this method, $\Delta F$ is calculated by canonical ensemble simulation, while $\langle V\rangle_{S}$ and $\langle V\rangle_{L}$ are calculated by separate isothermalisobaric simulations. Since the system one can simulate is not sufficiently large to ensure the equivalence of ensembles, such a method, in principle, could introduce some degree of uncertainty. In this work, we present a method of calculating $\Delta G$ directly by thermodynamic integration. 
The outline of the paper is as follows. In Sec. II A, we describe the thermodynamic integration method presented in Ref. [4] to calculate $\Delta F$ at a given temperature $T$ and pressure $P$. Section II B describes our adaptation of this method to calculate $\Delta G$ directly. In Sec. II C, we explain the need to control the translational degrees of freedom along the path for calculating $\Delta G$. Two approaches will be presented in Secs. II D and II E along with the corrections needed to account for the change in the free energy arising from the constraints. We then present results for the $\Delta G$ value and the melting temperature for truncated and shifted Lennard-Jones system in Sec. III, and conclude with a brief summary in Sec. IV.

\section{METHODOLOGY}

\section{A. Calculation of $\Delta F$}

The method developed by Grochola [4] differs from the conventional approaches such as the Einstein crystal method in that the thermodynamic path directly connects liquid and solid phases at given $T, P$, and $N$ in a reversible fashion. Thus, there is no need to introduce a reference system with known free energy. However, it still focuses on $\Delta F$ and all of the states along the path are subjected to constant $(T, V, N)$ simulation. The system volume $V$ must be determined based on $\langle V\rangle_{L}$ and $\langle V\rangle_{S}$, which in turn are obtained by separate isothermal-isobaric simulations at the desired values of $T, P$, and $N$. The integration path is divided into three pieces.

In the first stage of the path, the liquid state is converted into a high-density fluid state by linearly reducing the strength of the interaction potential while simultaneously changing linearly the length of the cubic simulation box from $L=\langle V\rangle_{L}^{1 / 3}$ to $L+B=\langle V\rangle_{S}^{1 / 3}$ so that the length of the simulation box for the first stage is given by $L+\lambda_{1} B$. The potential energy of the states along the path is given by

$$
\phi_{1}\left(\lambda_{1}\right)=\left(1-\eta \lambda_{1}\right) U
$$

where $\eta$ is a constant controlling the extent to which the strength of the interaction potential is reduced. The Helmholtz free energy difference for the first stage is given by

$$
\begin{aligned}
\Delta F_{1} & =\int_{0}^{1} d \lambda_{1}\left\langle\frac{\partial \phi_{1}}{\partial \lambda_{1}}\right\rangle_{\lambda_{1}} \\
& =\int_{0}^{1} d \lambda_{1}\left[-3 B\left(L+B \lambda_{1}\right)^{2} P_{v}\left(\lambda_{1}\right)-\eta\langle U\rangle_{\lambda_{1}}\right],
\end{aligned}
$$

where $\langle\ldots\rangle_{\lambda_{1}}$ indicates the canonical ensemble average taken for the system with interaction potential $\phi_{1}\left(\lambda_{1}\right)$ and $P_{v}$ is the virial pressure defined by

$$
P_{v}\left(\lambda_{1}\right)=\frac{k_{B} T N}{V\left(\lambda_{1}\right)}-\left\langle\frac{\partial \phi_{1}}{\partial V}\right\rangle_{\lambda_{1}},
$$

where $k_{B}$ is Boltzmann's constant.

In the second stage, the external potential consisting of Gaussian wells placed at the solid lattice points is gradually turned on while maintaining the interaction potential and the system volume constant at $\phi_{1}(1)=(1-\eta) U$ and $(L+B)^{3}$, re- spectively. This converts the high density fluid state into a structured low pressure solidlike state. In order for this process to be reversible, it is necessary to allow each potential well to interact with all of the particles within a certain cutoff distance. In other words, the influence of the potential well cannot be limited to a particular particle. As we shall see later, this has an important consequence when controlling the translational degrees of freedom of the system in simulation. The total potential energy for the second stage is given by

$$
\phi_{2}\left(\lambda_{2}\right)=(1-\eta) U+\lambda_{2} U_{\text {ext }},
$$

where $U_{e x t}=\sum_{i=1}^{N} \Sigma_{k} a \exp \left(-b r_{i k}^{2}\right)$ is the external potential, $r_{i k}$ is the distance between the $i$ th particle and $k$ th well, and the summation with respect to $k$ is taken over all Gaussian potential wells within a certain cutoff distance of the $i$ th particle. The cutoff distance reduces the computation required to calculate the external potential and should be chosen so that the effect of the Gaussian well on a particle is negligible beyond this distance. The Helmholtz free energy change for the second stage is given by

$$
\Delta F_{2}=\int_{0}^{1} d \lambda_{2}\left\langle U_{e x t}\right\rangle_{\lambda_{2}} .
$$

In the third stage of the integration, the strength of the interaction potential is increased to its full strength while gradually turning off the Gaussian external potential. The volume of the simulation box is again kept constant in the third stage. This converts the low-pressure solidlike phase into the desired solid phase. The total potential energy in the third stage is thus given by

$$
\phi_{3}\left(\lambda_{3}\right)=\left[(1-\eta)+\lambda_{3} \eta\right] U+\left(1-\lambda_{3}\right) U_{e x t},
$$

for which the Helmholtz free energy difference is

$$
\Delta F_{3}=\int_{0}^{1} d \lambda_{3}\left\langle\eta U-U_{e x t}\right\rangle_{\lambda_{3}} .
$$

Adding the contribution from each stage, we finally obtain

$$
\Delta F=\Delta F_{1}+\Delta F_{2}+\Delta F_{3}
$$

\section{B. Direct calculation of $\Delta G$}

The method just outlined is designed to calculate $\Delta F$, and each state along the reversible path is subjected to a canonical ensemble simulation. Instead, we propose to calculate $\Delta G$ directly by carrying out isothermal-isobaric simulation. To prevent the high-density fluid from evaporating during the first and second stages, we impose a constraint on the maximum possible volume of the system. This maximum volume $V_{m}$ is chosen to be sufficiently larger than the average volume of the starting liquid phase and the final solid phase so that the free energy of either phase will not be affected. Although the constraint is relevant only for the states near the end of the first stage and the beginning of the second stage, we maintain the constraint throughout the entire reversible path in order to avoid unnecessary complication in formulating the expression for $\Delta G$. At each stage, we use the same expressions for $\phi_{i}$ as before and arrive at 


$$
\begin{gathered}
\Delta G_{1}=\int_{0}^{1} d \lambda_{1}\langle-\eta U\rangle_{\lambda_{1}}, \\
\Delta G_{2}=\int_{0}^{1} d \lambda_{2}\left\langle U_{e x t}\right\rangle_{\lambda_{2}},
\end{gathered}
$$

and

$$
\Delta G_{3}=\int_{0}^{1} d \lambda_{3}\left\langle\eta U-U_{e x t}\right\rangle_{\lambda_{3}}
$$

where the thermal averages are calculated in the isothermalisobaric ensemble.

\section{Constraint on the translational degrees of freedom}

Near the end of the third stage, where $\lambda_{3}$ is close to unity, the integrand in Eq. (13), denoted hereafter by $g_{3}\left(\lambda_{3}\right)$, changes sharply with $\lambda_{3}$. At the same time, the accurate evaluation of $g_{3}\left(\lambda_{3}\right)$ becomes increasingly difficult since the contribution of $U_{\text {ext }}$ to $\phi_{3}$ approaches zero in this limit, and hence the system is free to move as a whole, causing the value of $U_{\text {ext }}$ to change wildly during the simulation [1]. Consequently, the statistical uncertainty in the evaluation of $g_{3}\left(\lambda_{3}\right)$ increases very rapidly as $\lambda_{3}$ approaches unity. This problem can be circumvented by constraining the translational degrees of freedom of the system, for which we propose two distinct methods and obtain the expression for the correction term to $\Delta G$ due to each constraint.

\section{Fixed particle constraint}

In the first method, we fix one of the particles in the system in space. In order that this constraint be consistent with the isotropic expansion and contraction of the box during an isothermal-isobaric simulation, the particle is placed at the origin of the system. This constraint is imposed throughout the three-stage reversible path and hence the thermodynamic integration gives the free energy difference between the constrained liquid and the solid states:

$$
\Delta G^{c p}=G_{S}^{c p}-G_{L}^{c p}=\Delta G_{1}^{c p}+\Delta G_{2}^{c p}+\Delta G_{3}^{c p},
$$

where the superscript $c p$ was used to indicate the quantities obtained in the constrained particle simulation. However, $\Delta G^{c p} \neq \Delta G$ and we must evaluate the correction term to estimate $\Delta G$ from $\Delta G^{c p}$.

For the liquid phase,

$$
G_{L}^{c p}-G_{L}=-k_{B} T \ln \left(\frac{Y_{L}^{c p}}{Y_{L}}\right)
$$

where

$$
\begin{aligned}
Y_{L}^{c p}= & \frac{l^{3}}{\Lambda^{3 N} N !} \int d V e^{-P V / k_{B} T} \theta\left(V_{m}-V\right) \int d \mathbf{r}_{1} \cdots d \mathbf{r}_{N} \delta\left(\mathbf{r}_{1}\right) \\
& \times \exp \left[-U\left(\mathbf{r}_{1}, \ldots, \mathbf{r}_{N}\right) / k_{B} T\right]
\end{aligned}
$$

and

$$
\begin{aligned}
Y_{L}= & \frac{1}{\Lambda^{3 N} N !} \int d V e^{-P V / k_{B} T} \theta\left(V_{m}-V\right) \int d \mathbf{r}_{1} \cdots d \mathbf{r}_{N} \\
& \times \exp \left[-U\left(\mathbf{r}_{1}, \ldots, \mathbf{r}_{N}\right) / k_{B} T\right]
\end{aligned}
$$

are, respectively, the partition functions for the constrained and unconstrained liquid phases, and $\Lambda$ is the thermal wavelength. The arbitrary length scale $l$ in Eq. (16) is introduced to make the equation dimensionally consistent but it does not affect the final results. $\theta$ is the step function defined by

$$
\theta(x)=\left\{\begin{array}{cc}
0, & x<0 \\
1 & \text { otherwise }
\end{array}\right.
$$

and does not allow the system volume $V$ to increase beyond a certain maximum value $V_{m}$. Equation (16) can be rewritten as

$$
\begin{aligned}
Y_{L}^{c p}= & \frac{l^{3}}{\Lambda^{3 N} N !} \int d V e^{-P V / k_{B} T} \theta\left(V_{m}-V\right) \int d \mathbf{r}_{2} \cdots d \mathbf{r}_{N} \\
& \times \exp \left[-U\left(\mathbf{0}, \mathbf{r}_{2}, \ldots, \mathbf{r}_{N}\right) / k_{B} T\right] \\
= & \frac{l^{3}}{\Lambda^{3 N} N !} \int d V e^{-P V / k_{B} T} \theta\left(V_{m}-V\right) \int \frac{d \mathbf{r}_{1}}{V} \int d \mathbf{r}_{2} \cdots d \mathbf{r}_{N} \\
& \times \exp \left[-U\left(\mathbf{0}, \mathbf{r}_{2}, \ldots, \mathbf{r}_{N}\right) / k_{B} T\right] \\
= & \frac{l^{3}}{\Lambda^{3 N} N !} \int d V e^{-P V / k_{B} T} \theta\left(V_{m}-V\right) \int \frac{d \mathbf{r}_{1}}{V} \int d \mathbf{r}_{2} \cdots d \mathbf{r}_{N} \\
& \times \exp \left[-U\left(\mathbf{r}_{1}, \mathbf{r}_{2}, \ldots, \mathbf{r}_{N}\right) / k_{B} T\right]
\end{aligned}
$$

where we made use of the fact that $U$ is translationally invariant under periodic boundary conditions. From Eqs. (15), (17), and (19), we find

$$
G_{L}^{c p}-G_{L}=-k_{B} T \ln \left(\left\langle\frac{l^{3}}{V}\right\rangle_{L}\right)
$$

where the ensemble average in Eq. (20) is evaluated by a liquid phase simulation with no constraint on the position of the first particle. Similarly,

$$
G_{S}^{c p}-G_{S}=-k_{B} T \ln \left(\left\langle\frac{l^{3}}{V}\right\rangle_{S}\right) \text {. }
$$

Thus, the desired free energy difference $\Delta G$ is

$$
\Delta G=G_{S}-G_{L}=G_{S}^{c p}-G_{L}^{c p}+k_{B} T \ln \left(\frac{\langle\rho\rangle_{S}}{\langle\rho\rangle_{L}}\right),
$$

where we used $\langle 1 / V\rangle=\langle\rho\rangle / N$. We identify the third term on the right hand side as the correction term to account for the fixed particle constraint. Note that the first two terms are of the order of $N$, while the third term is independent of $N$. Nonetheless, the term can be important if there is a large difference between $\langle\rho\rangle_{S}$ and $\langle\rho\rangle_{L}$ or if $N$ is not sufficiently large.

\section{E. Center of mass constraint}

Alternatively, one can control the translational motion of the system as a whole either by fixing the center of mass $\mathbf{r}_{\mathrm{c} . \mathrm{m}}$. 
of the system in space $[1,2,4]$ or by confining it to a small region in space [3]. However, Frenkel's method of fixing the center of mass as given in Refs. $[1,2]$ becomes prohibitively expensive when implemented in our case. In Frenkel's approach, which was implemented for Monte Carlo (MC) simulation, each attempted Monte Carlo move of a particle is accompanied by a linear translation of $N-1$ particles in order to fix $\mathbf{r}_{\mathrm{c} . \mathrm{m} \text {. }}$. However, as explained earlier, each Gaussian well must act on all of the particles in our technique, and hence Frenkel's method would require that the total external potential on the system be calculated at every attempted particle displacement during a Monte Carlo simulation. On the other hand, the approach presented in Ref. [3] remains computationally efficient to use in conjunction with our method and hence we use this approach. In particular, we constrain the center of mass $\mathbf{r}_{\mathrm{c} . \mathrm{m} \text {. }}$ of the system to a spherical region of a certain radius $R$ around the origin.

We impose the constraint on $\mathbf{r}_{\mathrm{c} . \mathrm{m}}$. on the states from the beginning of the second stage till the end of the third stage, which yields the free energy difference between the constrained states

$$
G_{S}^{\text {c.m. }}-G_{F}^{\text {c.m. }}=\Delta G_{2}^{\text {c.m. }}+\Delta G_{3}^{\text {c.m. },}
$$

where the subscript $F$ denotes the state at the beginning of the second stage, the subscript $S$ denotes, as before, the solid phase achieved at the end of the third stage, and finally, the superscript c.m. denotes the constraint on the center of mass. Note that

$$
G_{F}^{\text {c.m. }}-G_{F}=-k_{B} T \ln \left(\frac{Y_{F}^{\text {c.m. }}}{Y_{F}}\right),
$$

where

$$
\begin{aligned}
Y_{F}^{\text {c.m. }}= & \frac{1}{\Lambda^{3 N} N !} \int d V e^{-P V / k_{B} T} \theta\left(V_{m}-V\right) \int d \mathbf{r}_{1} \cdots d \mathbf{r}_{N} \\
& \times \theta\left(R-r_{\text {c.m. }}\right) \exp \left[-\phi_{2}(0) / k_{B} T\right]
\end{aligned}
$$

and

$$
\begin{aligned}
Y_{F}= & \frac{1}{\Lambda^{3 N} N !} \int d V e^{-P V / k_{B} T} \theta\left(V_{m}-V\right) \int d \mathbf{r}_{1} \cdots d \mathbf{r}_{N} \\
& \times \exp \left[-\phi_{2}(0) / k_{B} T\right]
\end{aligned}
$$

are, respectively, the partition functions of the constrained and the unconstrained $F$ states. From Eqs. (24)-(26), we have

$$
G_{F}^{\text {c.m. }}-G_{F}=-k_{B} T \ln \left[\left\langle\theta\left(R-r_{\text {c.m. } . ~}\right)\right\rangle_{\lambda_{2}=0}\right],
$$

where the ensemble average is recognized as the probability $p\left(r_{\text {c.m. }} \leqslant R\right)$ that the center of mass is within the spherical region of radius $R$ in a simulation carried out without the constraints on the center of mass. Similarly,

$$
G_{S}^{\text {c.m. }}-G_{S}=-k_{B} T \ln \left[\left\langle\theta\left(R-r_{\text {c.m. }}\right)\right\rangle_{\lambda_{3}=1}\right] .
$$

Using Eqs. (23), (27), and (28) and noting that $\Delta G_{1}=G_{F}-G_{L}$, we obtain

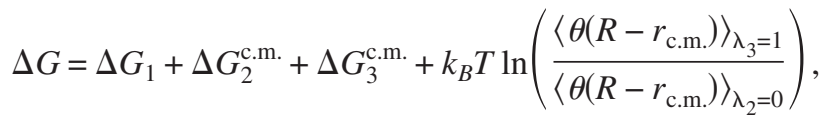

where the fourth term on the right hand side is the correction term to account for the constraint on the center of mass of the system.

In imposing the constraint on $\mathbf{r}_{\mathrm{c} . \mathrm{m}}$, it is important to consider how we define $\mathbf{r}_{\text {c.m. }}$. In the Einstein crystal method, if a particle leaves the box during the simulation, the particle should not be put back into the original simulation box on the other side of the box [1] in order to avoid a sudden change in the external potential. Then, $\mathbf{r}_{\text {c.m. }}$ is defined based on the instantaneous coordinates of the particles calculated under this convention. In the Einstein crystal method, the reversible path involves the solid phase at one end and the Einstein crystal at the other end. In the solid phase, particles typically vibrate about their average lattice positions and only rarely diffuse away from their initial positions. The same holds true for the states along the reversible path, since each particle is bound to the corresponding lattice point by a harmonic spring. As such, the definition mentioned above is quite appropriate. In our method, however, the reversible path involves fluid phase at one end and the solid phase at the other end. The above argument does not hold for the fluid phase and $\mathbf{r}_{\mathrm{c} . \mathrm{m} \text {. cannot be defined in the above mentioned }}$ fashion. The problem could be alleviated somewhat by imposing the constraint on $\mathbf{r}_{\text {c.m. }}$. only for the third stage of the thermodynamic integration. However, since a Gaussian well in our approach acts on all of the particles within a certain cutoff radius, the possibility of a given particle making an excursion over a long distance cannot be eliminated completely. For this reason, it is more appropriate, for our thermodynamic integration path, to define $\mathbf{r}_{\mathrm{c} . \mathrm{m} \text {. }}$ based on the instantaneous coordinates of the particles calculated under the usual convention that whenever a particle moves out of the simulation box, it is put back into the original simulation box on the other side of the box. The constraint on $\mathbf{r}_{\text {c.m. }}$ affects the system differently depending on how one defines $\mathbf{r}_{\mathrm{c} . \mathrm{m}}$, and hence the corresponding correction factor also depends on this definition, as we shall discuss in Sec. III.

\section{RESULTS AND DISCUSSION}

By means of Monte Carlo simulation, we calculated $\Delta G$ and the melting temperature of the truncated and shifted Lennard-Jones potential with the cutoff radius $r_{\text {cut }}=2.5 \sigma$ using the method described in the previous section. In what follows, the quantities with a superscript $*$ are in reduced units in terms of Lennard-Jones parameters $\sigma$ and $\epsilon$. We used a cubic simulation box with periodic boundary conditions. Following Ref. [4], the parameter $\eta$ was set to 0.9. The Gaussian wells were placed at the fcc lattice points and this external potential grid was made to expand and contract isotropically along with the simulation box during the volume change moves. The values for the parameters $a$ and $b$ of the Gaussian well were taken from Ref. [4] and are given by $a=-8.0 \epsilon$ and $b=6.93488 \sigma^{-2}$. These values were optimized 
in Ref. [4] at $T^{*}=0.7$ and $P^{*}=1.0$, and hence we evaluated $\Delta G$ at this condition. The cutoff distance for the Gaussian wells was chosen to be $2.5 \sigma$. The value of $V_{m}$ was determined so that the probability that $V$ exceeds $V_{m}$ in an unconstrained simulation of the liquid and the solid phase is negligible. In our simulation at $T^{*}=0.7$ and $P^{*}=1.0$, we chose $N / V_{m}=0.84 \sigma^{-3}$, for which this probability is only about 0.001 for the liquid phase. In terms of the effect on the Gibbs free energy, this amounts to $0.001 k_{B} T$, which is negligible for our purpose. For the solid phase, the effect is even smaller.

In the first stage of the reversible path, we started with a random configuration while in the second and the third stages, we started with a perfect fcc configuration. For each of the states along the reversible path, the system was equilibrated for $50000 \mathrm{MC}$ cycles followed by a production run of $2 \times 10^{5}$ cycles with block average taken over every 500 cycles. Each MC cycle consisted on average of two attempted volume change moves and one attempted displacement per particle. The magnitude of all attempted changes were adjusted during the equilibration period to give approximately $50 \%$ acceptance ratio. The statistical error was determined based on the block averages [10].

The integrals appearing in the expression for the Gibbs free energy difference were calculated by performing a trapezoidal rule integration with about 30 integrand evaluations for each stage. To see the effect of further decreasing the step size $h$ for each stage, we extrapolated the corresponding $\Delta G_{i}$ value, regarded as a linear function of $h^{2}$ [11], to the zero step size limit. This indicated that if we further decrease the step size for each stage, it would affect the $\Delta G$ value by less than $k_{B} T$, and hence we did not attempt it.

In the case of the c.m. constraint, the correction term involves the ensemble average of the step function, which is recognized as the probability $p\left(r_{\text {c.m. }} \leqslant R\right)$ that the center of mass is within the spherical region of a given radius $R$. As mentioned in Sec. II E, $\mathbf{r}_{\mathrm{c} . \mathrm{m}}$. can be defined in two different ways and the corresponding value of $p\left(r_{\mathrm{c} . \mathrm{m} .} \leqslant R\right)$ will depend on the definition. If we follow the definition of $\mathbf{r}_{\text {c.m. }}$ as used in the Einstein crystal method, the probability density of the center of mass will be uniform for the solid phase and will be equal to reciprocal volume of the Wigner-Seitz cell [1]. Thus, $p\left(r_{\text {c.m. }} \leqslant R\right)$ can be computed analytically in terms of the average density for the solid phase. However, we used the alternate definition for $\mathbf{r}_{\text {c.m. }}$ as given in Sec. II E, and the probability distribution of center of mass, thus defined, cannot be uniform as it is affected by the boundaries of the simulation box. For example, the probability density of finding $\mathbf{r}_{\mathrm{c} . \mathrm{m} \text {. }}$ near the corner of the box will be much smaller than that of finding $\mathbf{r}_{\text {c.m. }}$ at the center, since in the former case, the particles would have to be squeezed in the corner of the box and this configuration is energetically unfavorable at liquid or solidlike densities. Therefore, we calculated $p\left(r_{\mathrm{c} . \mathrm{m} .} \leqslant R\right)$ by a direct simulation for both the fluid phase $\left(\lambda_{2}=0\right)$ and the solid phase $\left(\lambda_{3}=1\right)$. In this work, we sampled $p\left(r_{\text {c.m. }} \leqslant R\right)$ for $2.2 \times 10^{6} \mathrm{MC}$ cycles for all values of $R$, following an equilibration period of 50000 cycles.

We present the $\Delta G$ values with various constraints in Fig. 1. It can be seen from the figure that the predicted $\Delta G$ for various values of $R$, with the correction, agree within the

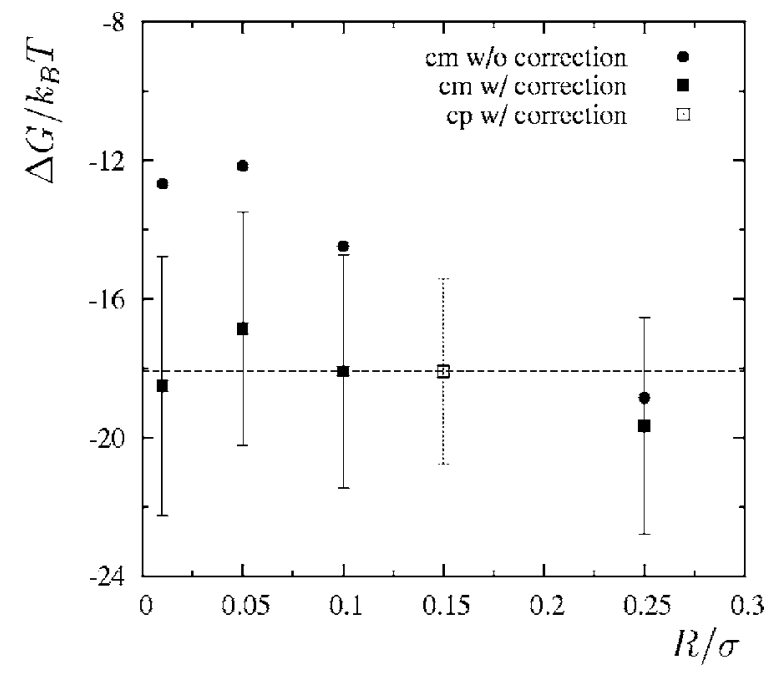

FIG. 1. Gibbs free energy difference $\Delta G=\left(G_{f c c}-G_{l i q}\right)$ between the fcc solid and the liquid at $P^{*}=1.0, T^{*}=0.7$, and $N=864$. The value obtained in Ref. [4] under the same condition is $\Delta G / k_{B} T$ $\simeq-1.4$. $R$ is the radius of the spherical region to which the center of mass of the system is confined. The open square symbol indicates the result for the fixed particle $(c p)$ constraint. Note that the value of $R$ is irrelevant for this method. For clarity, the error bars for uncorrected c.m. results are not shown. However, these are roughly of the same size as the corrected $c p$ result. The horizontal dashed line is drawn as a guide to the eyes.

statistical uncertainty. The accuracy in evaluating $\Delta G$ for various constraints depends on two factors: (i) the accuracy of the integration procedure which mainly depends on the behavior of the integrand $g_{3}\left(\lambda_{3}\right)$ near $\lambda_{3}=1.0$, and (ii) the accuracy in computing the correction term which depends on the evaluation of $p\left(r_{\mathrm{c} . \mathrm{m} .} \leqslant R\right)$. For $R / \sigma=0.25$, the translational degrees of freedom are less constrained compared to smaller $R$ values. As a result, $g_{3}\left(\lambda_{3}\right)$ varies more rapidly for this constraint and hence we evaluated the integrand at a larger number of quadrature points near $\lambda_{3}=1.0$. For smaller values of $R$, integrand is better behaved. However, an accurate computation of the correction term by a direct simulation requires a larger number of sampling since $p\left(r_{\text {c.m. }} \leqslant R\right)$ is small. This indicates that in the c.m. constraint method, some intermediate value of $R$, e.g., $R / \sigma=0.05$ and 0.1 in our case, would be optimum in terms of computational time and accuracy. We also note that our uncorrected c.m. results for smaller $R$ values are closer to the $\Delta G$ value obtained in Ref. [4]. This is expected since the latter value was obtained with the center of mass fixed throughout the reversible path and was not corrected for this constraint. There is still a noticeable discrepancy, however, between our uncorrected results and that in Ref. [4] $\left(\Delta G / k_{B} T \simeq-1.4\right)$. This can be attributed to the fact that we used more than twice the number of integrand evaluations (cf. Figs. 4-6 of Ref. [4]) for each stage of the integration to calculate $\Delta G$.

The results from the fixed particle constraint method are also shown in Fig. 1. In this case, the correction term involves bulk densities of the solid and the liquid phases, and hence its evaluation is relatively easy. However, as in the case of the c.m. constraint with $R / \sigma=0.25$, the calculation of 


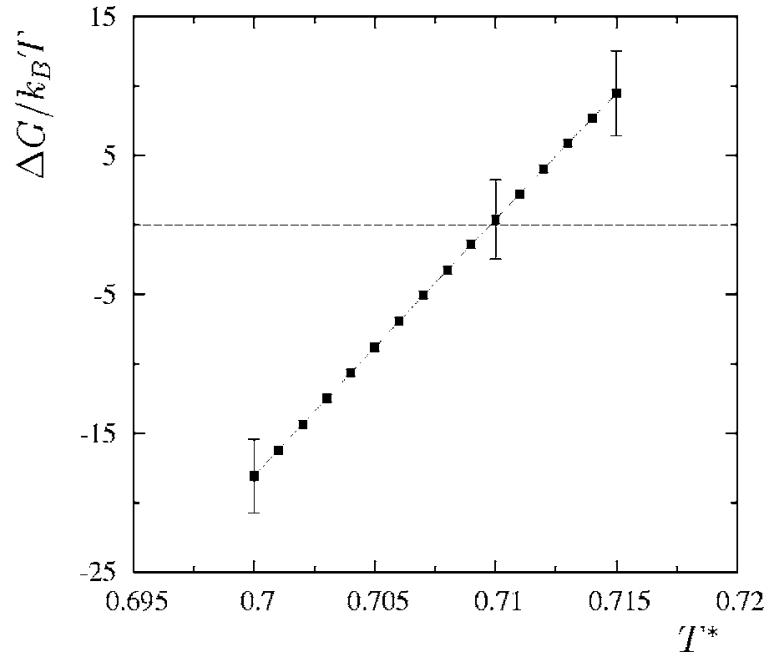

FIG. 2. Gibbs free energy difference $\Delta G$ as a function of $T^{*}$ at $P^{*}=1.0$ and $N=864$ for the fixed particle constraint. Note that the scale along the temperature axis is highly expanded. For clarity, the error bars are shown only for three points. For the rest of the points, the error bars are nearly of the same size.

$\Delta G$ required a larger number of $g_{3}\left(\lambda_{3}\right)$ evaluations than in the case of c.m. constraint with $R / \sigma \leqslant 0.1$. However, the fixed particle constraint is more effective in making $g_{3}\left(\lambda_{3}\right)$ smooth than the c.m. constraint with $R / \sigma=0.25$. We see from Fig. 1 that the $\Delta G$ value from the fixed particle constraint method agrees with the $\Delta G$ values from the c.m. constraint method within the statistical uncertainty.

To determine the melting temperature, we obtained the value of $\Delta G$ as a function of $T$ by integrating the relation

$$
k_{B} T^{2}\left(\frac{\partial\left(\Delta G / k_{B} T\right)}{\partial T}\right)_{P, N}=\left(\langle U\rangle_{L}+P\langle V\rangle_{L}\right)-\left(\langle U\rangle_{S}+P\langle V\rangle_{S}\right)
$$

with respect to $T$ while holding $P$ and $N$ constant and using the value of $\Delta G$ at $T^{*}=0.7$ as the initial condition. The melting temperature $T_{M}$ is then found by solving $\Delta G\left(T_{M}\right)=0$. The ensemble averages on the right hand side of Eq. (30) were evaluated by isothermal-isobaric simulations for each phase. The resulting plot for $\Delta G$ is shown in Fig. 2 for the fixed particle constraint. For the other constraints, the plot looks quite similar. The melting temperatures for various constraints obtained from these plots are listed in Table I. It can be seen from the table that the melting temperature predicted by the fixed particle constraint method agrees with those obtained by the c.m. constraint method for all values of $R$. Thus, the two methods we presented for treating the translational free energy are sufficiently accurate. It will also be observed that the effect of the correction term on the melting temperature is quite small under the conditions we studied. This is because the slope of the plot in Fig. 2 is large $\sim 1800$. Since the magnitude of the slope is directly proportional to
TABLE I. Effect of the correction term on melting temperatures for various constraints at $P^{*}=1.0$. The value of $\Delta G / k_{B} T \simeq-1.4$, as reported in Ref. [4], yields $T_{M}^{*}=0.701$.

\begin{tabular}{ccc}
\hline \hline Constraint & $T_{M}^{*}$ without correction & $T_{M}^{*}$ with correction \\
\hline c.m. $(R / \sigma=0.01)$ & $0.7068 \pm 0.0015$ & $0.7100 \pm 0.0022$ \\
c.m. $(R / \sigma=0.05)$ & $0.7065 \pm 0.0015$ & $0.7091 \pm 0.0020$ \\
c.m. $(R / \sigma=0.10)$ & $0.7078 \pm 0.0015$ & $0.7098 \pm 0.0020$ \\
c.m. $(R / \sigma=0.25)$ & $0.7102 \pm 0.0017$ & $0.7106 \pm 0.0019$ \\
Fixed particle $(c p)$ & $0.7098 \pm 0.0016$ & $0.7098 \pm 0.0016$ \\
\hline \hline
\end{tabular}

the enthalpy of fusion of the system [see Eq. (30)], $T_{M}$ will be more sensitive to the correction term as well as to the statistical uncertainty, when the enthalpy of fusion is small, in which case the accurate evaluation of the correction term as presented here becomes important. The correction term can also be significant if there is a large difference between the solid phase density and the liquid phase density or if the system size is small [see Eq. (22)].

The calculation of the correction term in the c.m. constraint method is more time consuming computationally than that in the fixed particle constraint. This is because, on the one hand, a small value of $R$ is required to ensure sufficient accuracy in evaluating $g_{3}\left(\lambda_{3}\right)$, which on the other hand implies that $p\left(r_{\mathrm{c} . \mathrm{m} .} \leqslant R\right)$ is small, requiring a large number of configurations to be generated for its accurate estimation. Overall, we found the fixed particle constraint method to be more efficient computationally even though $g_{3}\left(\lambda_{3}\right)$ is less well behaved as $\lambda_{3}$ approaches unity and hence more integrand evaluations are needed than the c.m. constraint method with smaller $R$ values.

\section{SUMMARY}

In this article, we presented two methods for treating consistently the translational free energy of the system while calculating directly the Gibbs free energy difference $\Delta G$ between the solid and the liquid phases at a given temperature and pressure. In particular, we have shown that both fixed particle constraint method and the center of mass constraint method are effective in controlling the translational degrees of freedom of the system and hence in reducing the statistical error in evaluating the integrand, while at the same time rendering the integrand itself well behaved. Thus, one of the major difficulties in evaluating melting point of a crystal can be circumvented by our methods. Finally, we note that our method extends naturally to binary systems [12].

\section{ACKNOWLEDGMENTS}

Acknowledgment is made to the Donors of the American Chemical Society Petroleum Research Fund for support. The computation reported here was made possible by a resource grant from the Ohio Supercomputer Center. 
[1] D. Frenkel and B. Smit, Understanding Molecular Simulation, 2nd ed. (Academic Press, San Diego, 2002).

[2] D. Frenkel and A. J. C. Ladd, J. Chem. Phys. 81, 3188 (1984).

[3] J. Q. Broughton and G. H. Gilmer, J. Chem. Phys. 79, 5095 (1983).

[4] G. Grochola, J. Chem. Phys. 120, 2122 (2004).

[5] W. G. Hoover and F. H. Ree, J. Chem. Phys. 49, 3609 (1968).

[6] N. B. Wilding and A. D. Bruce, Phys. Rev. Lett. 85, 5138 (2001).

[7] J. R. Errington, J. Chem. Phys. 120, 3130 (2004).
[8] D. M. Eike, J. F. Brennecke, and E. J. Maginn, J. Chem. Phys. 122, 014115 (2005).

[9] G. Grochola, I. K. Snook, and S. P. Russo, J. Chem. Phys. 122, 064711 (2005).

[10] M. P. Allen and D. J. Tildesley, Computer Simulation of Liquids (Oxford University Press, New York, 1987).

[11] W. H. Press, S. A. Teukolsky, W. T. Vetterling, and B. P. Flannery, Numerical Recepies (Cambridge University Press, Cambridge, U.K., 1992).

[12] P. A. Apte and I. Kusaka, J. Chem. Phys. 123, 194503 (2005). 